\title{
Research Article \\ Excited Neutrino Search Potential of the FCC-Based Electron-Hadron Colliders
}

\author{
A. Caliskan \\ Faculty of Engineering and Natural Sciences, Department of Physics Engineering, Gümüşhane University, 29100 Gümüşhane, Turkey \\ Correspondence should be addressed to A. Caliskan; acaliskan@gumushane.edu.tr
}

Received 20 October 2017; Accepted 9 December 2017; Published 31 December 2017

Academic Editor: Anna Cimmino

Copyright (C) 2017 A. Caliskan. This is an open access article distributed under the Creative Commons Attribution License, which permits unrestricted use, distribution, and reproduction in any medium, provided the original work is properly cited. The publication of this article was funded by SCOAP . $^{3}$

\begin{abstract}
The production potential of the excited neutrinos at the FCC-based electron-hadron colliders, namely, the ERL60 $\otimes$ FCC with $\sqrt{s}=$ $3.46 \mathrm{TeV}$, the ILC $\otimes \mathrm{FCC}$ with $\sqrt{s}=10 \mathrm{TeV}$, and the PWFA-LC $\otimes \mathrm{FCC}$ with $\sqrt{s}=31.6 \mathrm{TeV}$, has been analyzed. The branching ratios of the excited neutrinos have been calculated for the different decay channels and shown that the dominant channel is $\nu^{\star} \rightarrow e W^{+}$. We have calculated the production cross sections with the process of $e p \rightarrow v^{\star} q \rightarrow e W^{+} q$ and the decay widths of the excited neutrinos with the process of $\nu^{\star} \rightarrow e W^{+}$. The signals and corresponding backgrounds are studied in detail to obtain accessible mass limits. It is shown that the discovery limits obtained on the mass of the excited neutrino are $2452 \mathrm{GeV} \mathrm{for} L_{\text {int }}=100 \mathrm{fb}^{-1}$, $5635 \mathrm{GeV}$ for $L_{\text {int }}=10 \mathrm{fb}^{-1}\left(6460 \mathrm{GeV}\right.$ for $\left.L_{\text {int }}=100 \mathrm{fb}^{-1}\right)$, and $10200 \mathrm{GeV}$ for $L_{\text {int }}=1 \mathrm{fb}^{-1}\left(13960 \mathrm{GeV}\right.$ for $\left.L_{\text {int }}=10 \mathrm{fb}^{-1}\right)$, for the center-of-mass energies of $3.46,10$, and $31.6 \mathrm{TeV}$, respectively.
\end{abstract}

\section{Introduction}

The Standard Model (SM) of particle physics has so far been in agreement with the results of numerous experiments. The discovery of the Higgs boson [1] has also increased the reliability of the SM. However, there are some problems which have not been entirely solved by the SM, such as quark-lepton symmetry, family replication, number of families, fermion's masses and mixing pattern, and hierarchy problems. Several theories beyond the SM (BSM), including extra dimensions, supersymmetry (SUSY), and compositeness, have been proposed to solve these problems. The best way to explain the inflation of fundamental particles in the SM is to assume that they have more fundamental matter constituents. Therefore, a natural explanation for the replication of the SM fermionic families is lepton and quark compositeness, in which both matter and antimatter elementary particles have a substructure called preon [2]. The composite models have been characterized by an energy scale, namely, compositeness scale, $\Lambda$. A typical consequence of the compositeness is the appearance of excited leptons and quarks [3, 4]. Charged $\left(e^{\star}, \mu^{\star}, \tau^{\star}\right)$ and neutral $\left(v_{e}^{\star}, v_{\mu}^{\star}, v_{\tau}^{\star}\right)$ excited leptons are predicted by the composite models. The SM fermions are considered as ground states of a rich and heavier spectrum of the excited states. An excited spin-1/2 lepton is considered to be both the lowest radial and orbital excitation. Excited states with spin$3 / 2$ are also expected to exist [5].

No evidence for excited lepton production has been found so far in searches based on data samples collected by the LEP [6], HERA [7], Tevatron [8], CMS [9], and ATLAS [10] experiments. For the excited electron [11, 12], muon [13], and neutrino [14-17], there are some phenomenological studies at the future high-energy colliders.

Current experimental lower bounds on the masses of the excited neutrinos are $m_{\gamma^{\star}}>102.6 \mathrm{GeV}$ [6] from LEP-L3 collaboration (pair production) assuming $f=-f^{\prime}=1$, $m_{\gamma^{\star}}>213 \mathrm{GeV}$ [18] at 95\% CL from HERA-H1 collaboration (single production) assuming $f=f^{\prime}=1$ and $m_{\gamma^{\star}}>$ 1.6 TeV [18], namely, the strongest limit, from LHC-ATLAS collaboration (pair production) assuming $f=f^{\prime}=1$.

The Future Circular Collider (FCC) is a post-Large Hadron Collider (LHC) accelerator project [19], with $\sqrt{s}=$ $100 \mathrm{TeV}$, proposed at CERN and supported by European Union within the Horizon 2020 Framework Programme for Research and Innovation. Besides the $p p$ option, FCC also includes $e^{+} e^{-}$collider option (TLEP) at the same tunnel [20]. 
TABLE 1: Main parameters of the FCC-based ep colliders.

\begin{tabular}{lccc}
\hline Colliders & $E_{e}(\mathrm{TeV})$ & $\mathrm{CM}$ energy $(\mathrm{TeV})$ & $L_{\text {int }}\left(\mathrm{fb}^{-1}\right.$ per year $)$ \\
\hline ERL60 $\otimes$ FCC & 0.06 & 3.46 & 100 \\
ILC $\otimes$ FCC & 0.5 & 10 & $10-100$ \\
PWFA-LC $\otimes$ FCC & 5 & 31.6 & $1-10$ \\
\hline
\end{tabular}

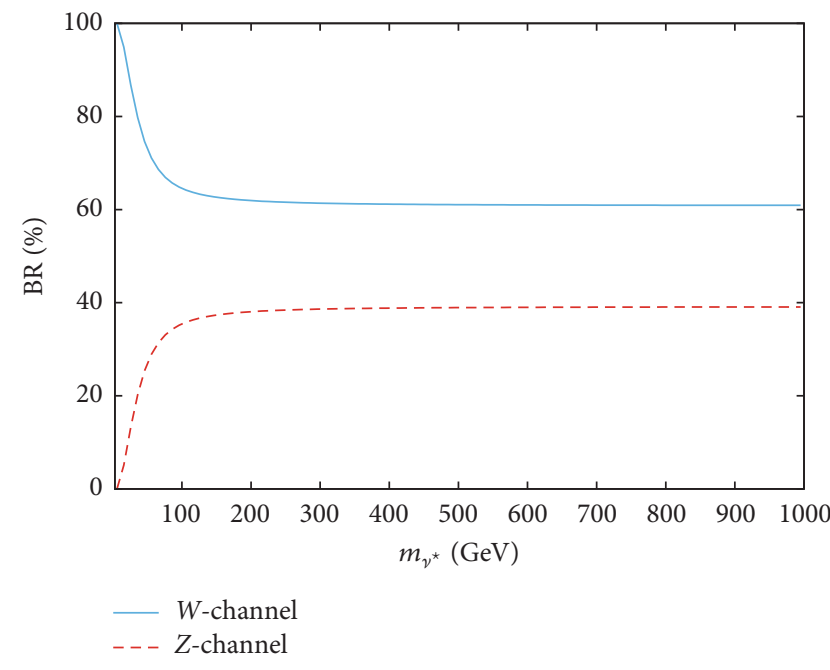

(a)

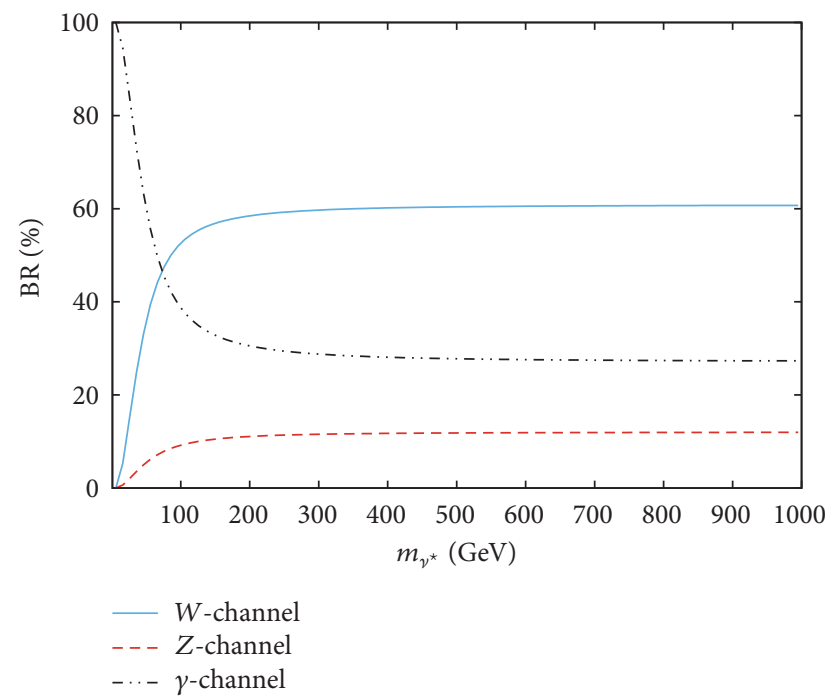

(b)

FIGURE 1: The branching ratios (\%) depending on the mass of the excited neutrino for $f=f^{\prime}=1$ (a) and $f=-f^{\prime}=1(\mathrm{~b})$.

Construction of the future $e^{+} e^{-}$and $\mu^{+} \mu^{-}$colliders tangential to the FCC will also provide several $e p$ and $\mu p$ collider options [21].

In this paper, we analyze the potential of the FCCbased ep colliders, namely, ERL60 $\otimes$ FCC, ILC $\otimes$ FCC, and PWFA-LC $\otimes$ FCC, for the excited neutrino searches. The ERL60 denotes energy recovery linac proposed for the LHeC main option [22] and can also be used for the FCC-based ep colliders. The ILC and the PWFA-LC mean International Linear Collider [23] and Plasma Wake Field Accelerator Linear Collider [24], respectively. The FCC-based ILC $\otimes$ FCC and PWFA-LC $\otimes$ FCC colliders have been proposed in [25]. Energy of the electron beams, center-of-mass energy, and luminosity values of the FCC-based ep colliders are presented in Table $1[25,26]$.

We introduce the effective Lagrangian, the decay widths, and the branching ratios of the excited neutrinos in Section 2. In Section 3, we analyze the signal and backgrounds for the process ep $\rightarrow v^{\star} q \rightarrow e W^{+} q$, and finally we summarize our results in Section 4.

\section{Production of the Excited Neutrinos}

The interaction between a spin-1/2 excited lepton, a gauge boson $\left(V=\gamma, Z, W^{ \pm}\right)$, and the SM leptons is described by $S U(2) \times U(1)$ invariant Lagrangian $[4,27,28]$ as

$$
L=\frac{1}{2 \Lambda} \overline{l_{R}^{*}} \sigma^{\mu \nu}\left[f g \frac{\vec{\tau}}{2} \cdot \vec{W}_{\mu \nu}+f^{\prime} g^{\prime} \frac{Y}{2} B_{\mu \nu}\right] l_{L}+\text { h.c. }
$$

where $\Lambda$ is the new physics scale responsible for the existence of the excited leptons, $\vec{W}_{\mu \nu}$ and $B_{\mu \nu}$ are the field strength tensors, $g$ and $g^{\prime}$ are the gauge couplings, $f$ and $f^{\prime}$ are the scaling factors for the gauge couplings of $S U(2)$ and $U(1), \sigma^{\mu \nu}=$ $i\left(\gamma^{\mu} \gamma^{\nu}-\gamma^{\nu} \gamma^{\mu}\right) / 2$ where $\gamma^{\mu}$ are the Dirac matrices, $\vec{\tau}$ denotes the Pauli matrices, and $Y$ is hypercharge.

For an excited neutrinos, three decay modes are possible: radiative decay $\nu^{\star} \rightarrow \nu \gamma$, neutral weak decay $\nu^{\star} \rightarrow \nu Z$, and charged weak decay $\nu^{\star} \rightarrow e W^{+}$. The branching ratios (BR) of the excited neutrino for the couplings $f=f^{\prime}=1$ and $f=-f^{\prime}=1$ are given in Figure 1 . One may note that the electromagnetic interaction between excited neutrino and ordinary neutrino, namely, $\gamma$-channel, vanishes for $f=f^{\prime}=$ 1. As clearly visible from Figure 1 , the $W$-channel, whose branching ratio is $\sim 60 \%$, is dominant in the whole mass range for $f=f^{\prime}=1$. In the case of $f=-f^{\prime}=1$, the branching ratio for the individual decay channels reaches the constant value of $60 \%$ for the $W$-channel, $12 \%$ for the $Z$-channel, and $28 \%$ for the $\gamma$-channel at higher neutrino masses $\left(m_{v^{\star}}>150 \mathrm{GeV}\right)$. Since the charged weak decay $\left(\nu^{\star} \rightarrow \mathrm{eW} W^{+}\right)$is dominant in both cases, we preferred this channel for investigating the excited neutrino in future linear collider experiments.

Neglecting the SM lepton mass, we find the decay width of excited leptons as

$$
\Gamma\left(l^{\star} \longrightarrow l V\right)=\frac{\alpha m^{\star 3}}{4 \Lambda^{2}} f_{V}^{2}\left(1-\frac{m_{V}^{2}}{m^{\star 2}}\right)^{2}\left(1+\frac{m_{V}^{2}}{2 m^{\star 2}}\right),
$$




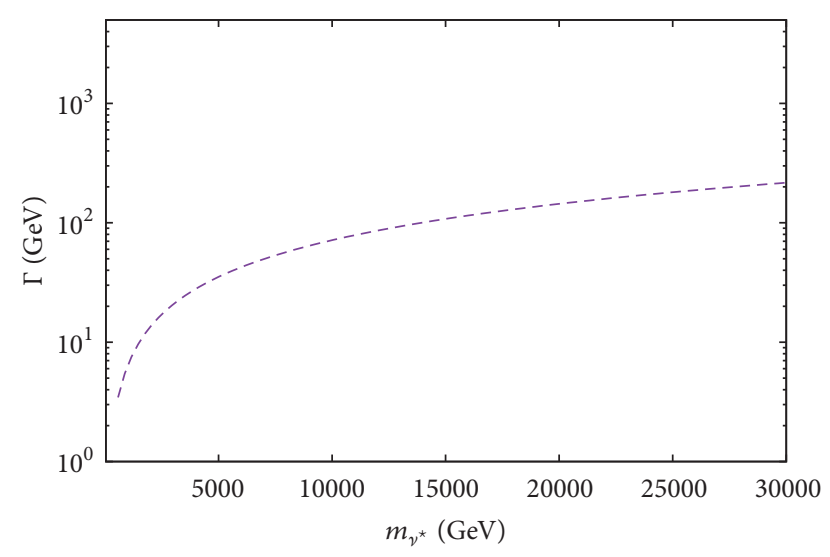

FIGURE 2: The total decay widths of the excited neutrino for the scale $\Lambda=m_{v^{\star}}$ and the coupling $f=f^{\prime}=1$.

where $f_{V}$ is the new electroweak coupling parameter corresponding to the gauge boson $V$, where $V=W^{+}, Z, \gamma$, and $f_{\gamma}=$ $\left(f-f^{\prime}\right) / 2, f_{Z}=\left(f \cot \theta_{W}+f^{\prime} \tan \theta_{W}\right) / 2, f_{W}=f / \sqrt{2} \sin \theta_{W}$, where $\theta_{W}$ is the weak mixing angle and $m_{V}$ is the mass of the gauge boson. The total decay widths of the excited neutrino for the scale $\Lambda=m_{v^{*}}$ is given in Figure 2 .

\section{Signal and Background Analysis}

We analyze the potentials of the future ep collider machines to search for the excited neutrinos via the single production reaction ep $\rightarrow v^{\star} X$ with subsequent decay of the excited neutrino into an electron and a $W^{+}$boson. Therefore, we consider the process $e p \rightarrow W^{+} e X$ and subprocesses $e q(\bar{q}) \rightarrow$ $W^{+} e q(\bar{q})$. The signal and background analysis were done at the parton level by using the high-energy simulation program CALCHEP in the version 3.6.25 [29]. We used the CTEQ6L [30] parton distribution functions in our calculations.

For a comparison of different FCC-based $e p$ colliders, the signal cross sections for excited neutrino production are presented in Figure 3, assuming the coupling parameter $f=$ $f^{\prime}=1$.

\subsection{ERL60 $\otimes$ FCC Collider. The ERL60 $\otimes$ FCC is a FCC-based} future ep collider with the center-of-mass energy of $3.46 \mathrm{TeV}$. Keeping in mind that the lower bound on the mass of the excited neutrino is $1.6 \mathrm{TeV}\left(m_{\gamma^{\star}}>1.6 \mathrm{TeV}\right)$, we have explored the mass limits for the discovery of the excited neutrinos in the range of 1.6 and $3.46 \mathrm{TeV}$ at the ERL60 $\otimes$ FCC collider. To separate signal from backgrounds, final state particles (electron, $W^{+}$boson and jets) with $p_{T}^{e, W, j}>20 \mathrm{GeV}$ are required. The SM cross section after the application of these cuts is $\sigma_{B}=3.96 \mathrm{pb}$. In order to define the kinematical cuts best suited for discovery, we have plotted the normalized transverse momentum and the normalized pseudorapidity distributions of the final state particles. Figure 4 shows the normalized $p_{T}$ distributions of the final state $W^{+}$bosons (a), pseudorapidity $(\eta)$ distributions of the final state electron (b), and the $\eta$ distributions of the final state $W^{+}$(c), for signals corresponding to excited neutrino masses of 1000 and

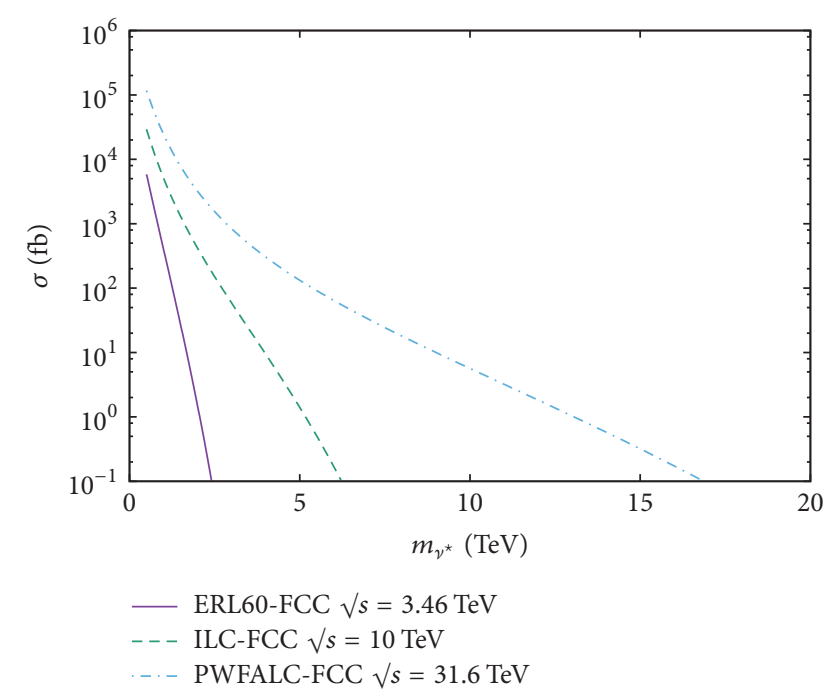

FIGURE 3: The total cross sections as a function of the excited neutrino mass at the ep colliders with various center-of-mass energies, assuming $f=f^{\prime}=1$ and $\Lambda=m_{v^{\star}}$.

$2000 \mathrm{GeV}$ and the SM backgrounds. The $p_{T}$ distributions of the final state electrons are the same as those of the final state $W^{+}$bosons. As can be seen from Figure 4, the kinematical cuts $p_{T}^{W, e}>200 \mathrm{GeV},-5<\eta^{e}<-1$ and $-4.5<\eta^{W}<-2$ drastically reduce the background while keeping the signal almost unchanged. The invariant mass distributions of the $e W^{+}$system after the application of all kinematical cuts is reported in Figure 5. The separation of the signal from the background improved.

A natural way of extracting the excited neutrino signal, and the same time suppressing the SM background is to impose a cut on the $\mathrm{eW}^{+}$invariant mass in addition to kinematical cuts. Therefore, we have selected events within the mass window $m_{v^{\star}}-2 \Gamma_{v^{\star}}<m_{e W}<m_{v^{\star}}+2 \Gamma_{v^{\star}}$.

We define statistical significance (SS) of the expected signal yield as

$$
\mathrm{SS}=\frac{\left|\sigma_{S+B}-\sigma_{B}\right|}{\sqrt{\sigma_{B}}} \sqrt{L_{\mathrm{int}}}
$$

where $\sigma_{S+B}$ denotes the cross section due to the excited neutrino production and the SM backgrounds, $\sigma_{B}$ denotes the SM cross section, and $L_{\text {int }}$ is the integrated luminosity of the collider. Assuming $f=f^{\prime}=1$ and $\Lambda=m_{v^{\star}}$, we have calculated the signal, the background cross sections, and SS in $\mathrm{eW}^{+}$invariant mass bins since the signal is concentrated in a small region proportional to the invariant mass resolution. The results are summarized in Table 2. The ERL60 $\otimes$ FCC collider can discover the excited neutrino in $v^{\star} \rightarrow W^{+} e$ decay mode for the coupling $f=f^{\prime}=1$ up to the mass of $2452 \mathrm{GeV}$ taking into account the discovery criterion SS $\geq 5$ (99\% CL).

3.2. ILC $\otimes$ FCC Collider. The ILC $\otimes$ FCC collider with the center-of-mass energy of $10 \mathrm{TeV}$ can search for the excited neutrino in a wider mass range compared to the ERL $60 \otimes$ FCC collider. We have explored the mass limits for discovery of 


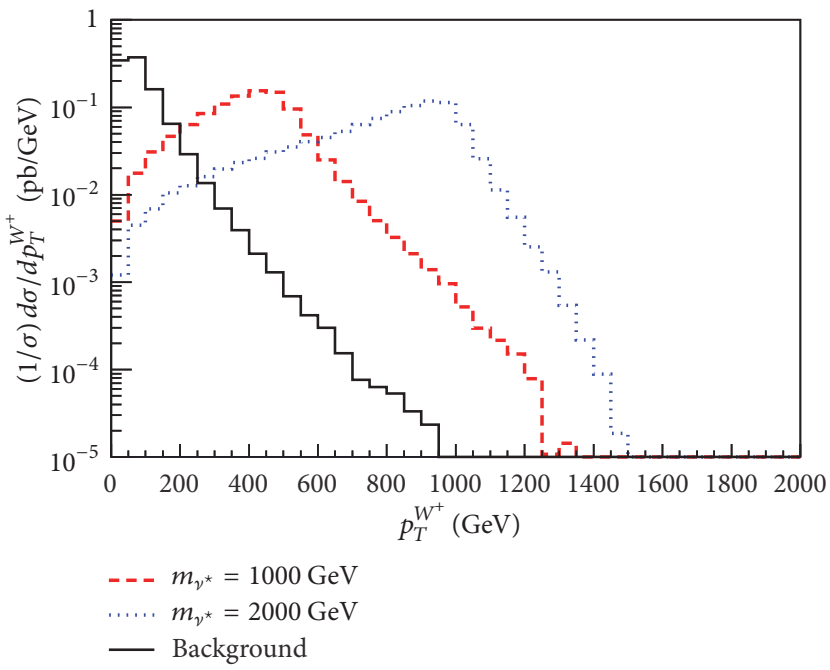

(a)

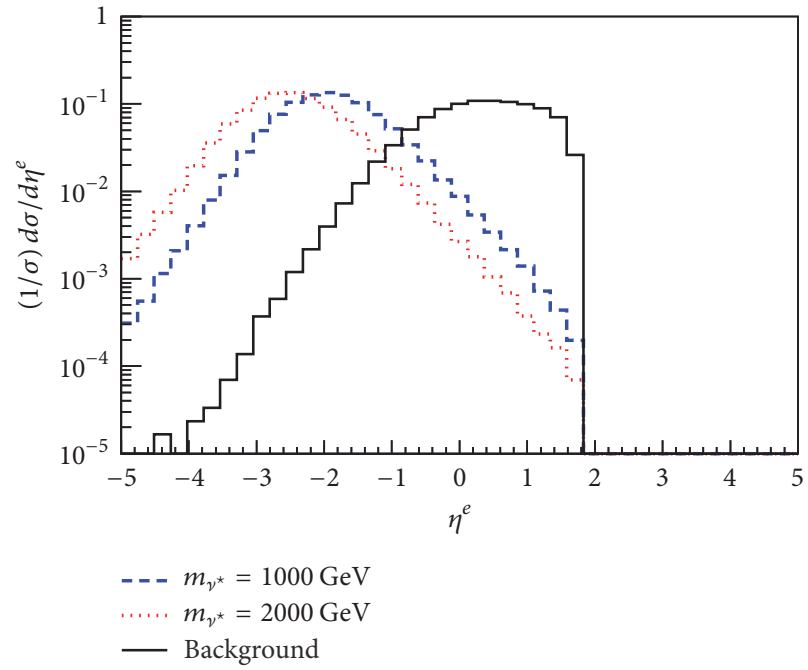

(b)

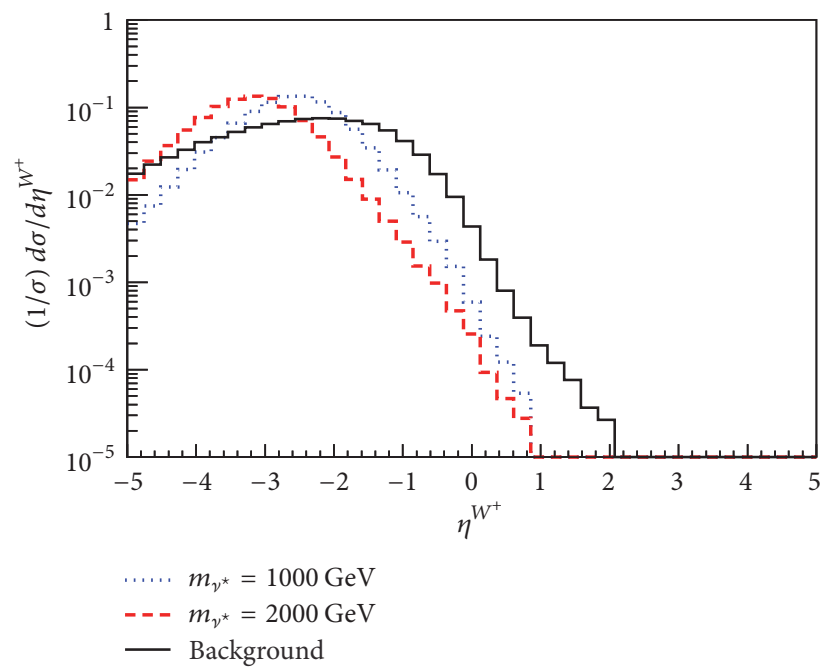

(c)

FIGURE 4: The normalized transverse momentum distributions of the final state $W^{+}$bosons (a), the normalized pseudorapidity distributions of the final state electrons (b), and the normalized pseudorapidity distributions of the final state $W^{+}$bosons (c) for $f=f^{\prime}=1$ and $\Lambda=m_{v^{\star}}$ at the ERL60 $\otimes$ FCC collider.

TABLE 2: The statistical significance (SS) values and the cross sections of the excited neutrino signal and relevant backgrounds at ERL60 $\otimes$ FCC collider with $\sqrt{s}=3.46 \mathrm{TeV}$ and $L_{\text {int }}=100 \mathrm{fb}^{-1}$, assuming $\Lambda=m_{\gamma^{\star}}$ and $f=f^{\prime}=1$.

\begin{tabular}{lllr}
\hline Mass $(\mathrm{GeV})$ & $\sigma_{S+B}(\mathrm{pb})$ & $\sigma_{B}(\mathrm{pb})$ & SS \\
\hline 1600 & $7.21 \times 10^{-3}$ & $1.86 \times 10^{-4}$ & 162.9 \\
1800 & $2.47 \times 10^{-3}$ & $9.60 \times 10^{-5}$ & 76.5 \\
2000 & $7.65 \times 10^{-4}$ & $4.15 \times 10^{-5}$ & 35.5 \\
2200 & $2.09 \times 10^{-4}$ & $1.49 \times 10^{-5}$ & 15.9 \\
2300 & $1.03 \times 10^{-4}$ & $8.48 \times 10^{-6}$ & 10.2 \\
2400 & $4.82 \times 10^{-5}$ & $4.64 \times 10^{-6}$ & 6.4 \\
2500 & $2.14 \times 10^{-5}$ & $2.41 \times 10^{-6}$ & 3.8 \\
2600 & $8.85 \times 10^{-6}$ & $1.19 \times 10^{-6}$ & 2.2 \\
2700 & $3.32 \times 10^{-6}$ & $5.41 \times 10^{-7}$ & 1.1 \\
\hline
\end{tabular}


TABLE 3: The statistical significance (SS) values and the cross sections of the excited neutrino signal and relevant background at the ILC $\otimes$ FCC collider with $\sqrt{s}=10 \mathrm{TeV}$ assuming the coupling $f=f^{\prime}=1$ and the energy scale $\Lambda=m_{v^{\star}}$.

\begin{tabular}{|c|c|c|c|c|}
\hline Mass $(\mathrm{GeV})$ & $\sigma_{B}(\mathrm{pb})$ & $\sigma_{S+B}(\mathrm{pb})$ & $\begin{array}{c}L_{\text {int }}=10 \mathrm{fb}^{-1} \\
\mathrm{SS}\end{array}$ & $\begin{array}{c}L_{\text {int }}=100 \mathrm{fb}^{-1} \\
\text { SS }\end{array}$ \\
\hline 2000 & $1.81 \times 10^{-3}$ & $1.47 \times 10^{-1}$ & 342.1 & 1081.9 \\
\hline 2500 & $1.24 \times 10^{-3}$ & $5.85 \times 10^{-2}$ & 162.9 & 515.1 \\
\hline 3000 & $7.37 \times 10^{-4}$ & $2.43 \times 10^{-2}$ & 86.8 & 274.7 \\
\hline 3500 & $3.94 \times 10^{-4}$ & $1.03 \times 10^{-2}$ & 49.6 & 157 \\
\hline 4000 & $1.85 \times 10^{-4}$ & $4.28 \times 10^{-3}$ & 30 & 95.1 \\
\hline 4500 & $8.27 \times 10^{-5}$ & $1.74 \times 10^{-3}$ & 18.1 & 57.4 \\
\hline 5000 & $3.58 \times 10^{-5}$ & $6.69 \times 10^{-4}$ & 10.5 & 33.4 \\
\hline 5500 & $1.47 \times 10^{-5}$ & $2.43 \times 10^{-4}$ & 5.9 & 18.8 \\
\hline 6000 & $6.07 \times 10^{-6}$ & $8.15 \times 10^{-5}$ & 3 & 9.6 \\
\hline 6500 & $2.26 \times 10^{-6}$ & $2.49 \times 10^{-5}$ & 1.5 & 4.7 \\
\hline 7000 & $7.83 \times 10^{-7}$ & $6.69 \times 10^{-6}$ & 0.6 & 2.1 \\
\hline 7500 & $2.37 \times 10^{-7}$ & $1.49 \times 10^{-6}$ & 0.2 & 0.8 \\
\hline
\end{tabular}

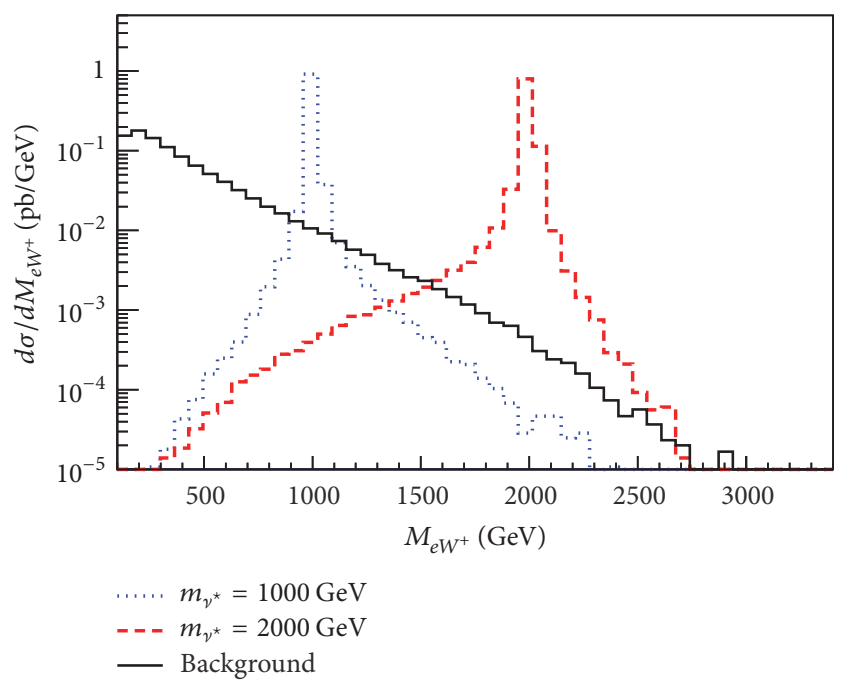

FIGURE 5: The invariant mass distributions of the excited neutrino signal and the corresponding background for $\Lambda=m_{v^{\star}}$ and $f=$ $f^{\prime}=1$ at the ERL60 $\otimes$ FCC collider.

the excited neutrinos in the mass range from 1.6 to $10 \mathrm{TeV}$. In order to separate the excited neutrino signals from the background we have required $p_{T}^{e, W, j}>20 \mathrm{GeV}$, as for the ERL60 $\otimes$ FCC collider. Subsequently, the SM background cross section for the ILC $\otimes$ FCC collider is found to be $\sigma_{B}=$ $15.74 \mathrm{pb}$. The normalized $p_{T}$ distributions of the final state electrons, the $\eta$ distributions of the final state $W^{+}$bosons, and the $\eta$ distributions of the final state electrons are presented in Figure 6. Also in this case, final state electrons and $W^{+}$ bosons have the same $p_{T}$ distribution. The kinematical cuts $p_{T}^{W, e}>200 \mathrm{GeV},-3.4<\eta^{W}<0.4$, and $-5<\eta^{e}<1$ are optimal for increasing the potential discovery. The invariant mass distributions of the $\mathrm{eW}^{+}$system after the application of all kinematical cuts is reported in Figure 7.
Signal and background cross sections in $\mathrm{eW}^{+}$invariant mass bins $m_{v^{\star}}-2 \Gamma_{v^{\star}}<m_{e W}<m_{v^{\star}}+2 \Gamma_{\nu^{\star}}$ and the SS values calculated for $L_{\text {int }}=10 \mathrm{fb}^{-1}$ and $L_{\text {int }}=100 \mathrm{fb}^{-1}$ are summarized in Table 3.

Assuming $f=f^{\prime}=1$ and $\Lambda=m_{v^{\star}}$, taking into account the calculated SS values for SS $\geq 5$ criterion, the ILC $\otimes$ FCC collider can probe the excited neutrino up to the masses of 5635 and $6460 \mathrm{GeV}$ for the integrated luminosities of $L_{\text {int }}=$ $10 \mathrm{fb}^{-1}$ and $L_{\text {int }}=100 \mathrm{fb}^{-1}$, respectively.

3.3. PWFA-LC $\otimes$ FCC Collider. If the excited neutrinos had not been observed at the ERL60 $\otimes$ FCC and the ILC $\otimes$ FCC colliders, they would have been explored up to the mass of 31.6 TeV at the PWFA-LC $\otimes$ FCC collider that has the widest research potential. We have explored the mass limits for discovering the excited neutrinos in a broad mass spectrum from 1.6 to $31.6 \mathrm{TeV}$. The SM background cross section is found to be $\sigma_{B}=58.15 \mathrm{pb}$ after the application of the same initial kinematical cuts. Figure 8 shows the $p_{T}$ distributions of the final state $W^{+}$bosons, the $\eta$ distributions of the final state electrons, and the $\eta$ distributions of the $W^{+}$boson for the excited neutrino masses of 5000, 10000, 15000, and $20000 \mathrm{GeV}$ versus the backgrounds. As already discussed, the $p_{T}$ distributions of the $W^{+}$bosons are the same for the final state electrons. By requiring $p_{T}^{W, e}>400 \mathrm{GeV}-5<\eta^{e}<2.5$, and $-2.5<\eta^{W}<1$, the background is suppressed, whereas the signal remains almost unchanged. The invariant mass distributions of the $\mathrm{eW}^{+}$system obtained after application of all cuts is reported in Figure 9. We have also required the $\mathrm{eW} \mathrm{W}^{+}$invariant masses to be in the range $m_{\gamma^{\star}}-2 \Gamma_{\gamma^{\star}}<m_{e W}<$ $m_{\gamma^{\star}}+2 \Gamma_{\nu^{\star}}$.

Assuming $f=f^{\prime}=1$ and $\Lambda=m_{v^{\star}}$, the signal and the background cross sections for PWFA-LC $\otimes$ FCC collider, as well as the SS values, are summarized in Table 4 for two integrated luminosity values, namely, $L_{\text {int }}=1 \mathrm{fb}^{-1}$ and $L_{\text {int }}=$ $10 \mathrm{fb}^{-1}$. For the energy scale of $\Lambda=m_{v^{\star}}$, the PWFA-LC $\otimes$ FCC collider can probe the excited neutrino up to the masses of 


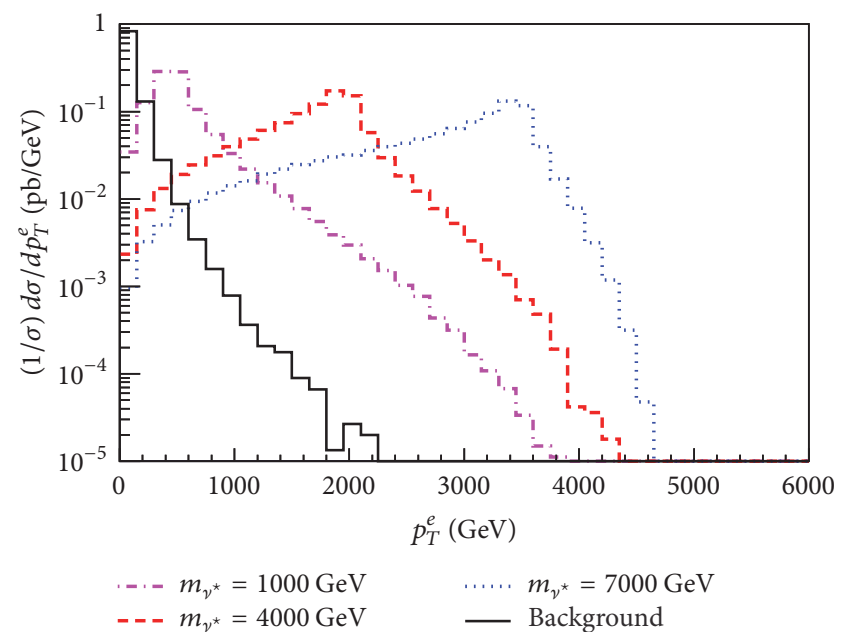

(a)

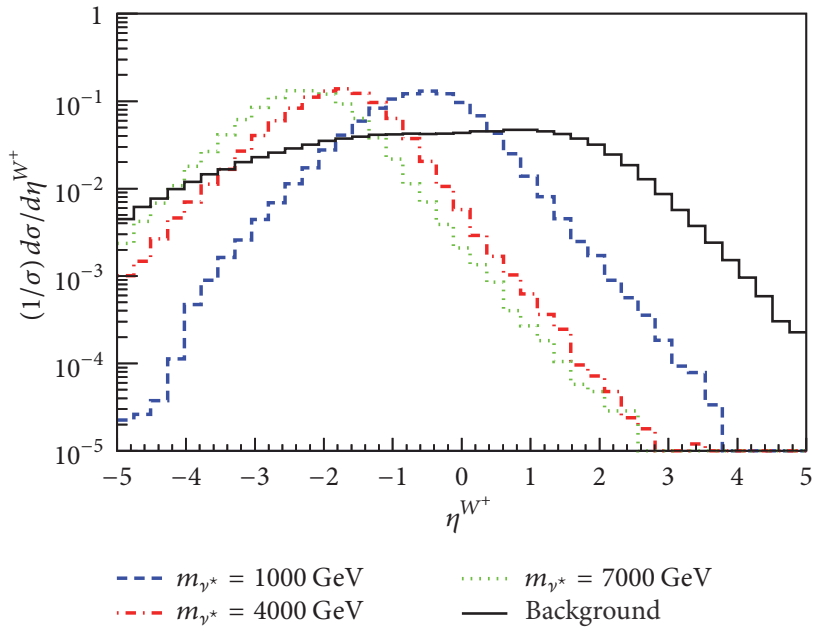

(b)

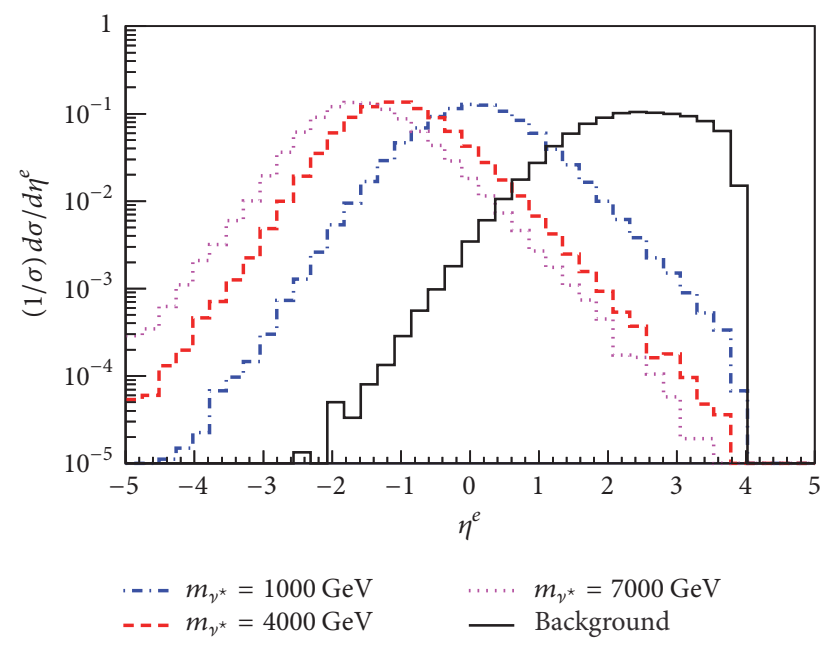

(c)

FIGURE 6: The normalized transverse momentum distributions of the final state electrons (a), the normalized pseudorapidity distributions of the final state $W^{+}$bosons (b), and the normalized pseudorapidity distributions of the final state electrons (c) for $f=f^{\prime}=1$ and $\Lambda=m_{v^{\star}}$ at the ILC $\otimes$ FCC collider.

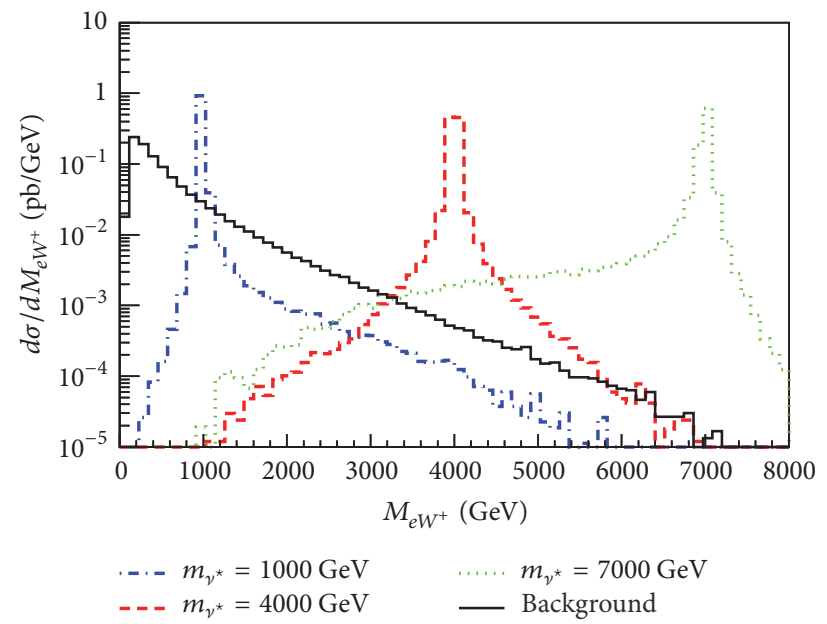

FIGURE 7: The invariant mass distributions of the excited neutrino signal and the corresponding background for $\Lambda=m_{\gamma^{\star}}$ and $f=f^{\prime}=1$ at the ILC $\otimes$ FCC collider. 


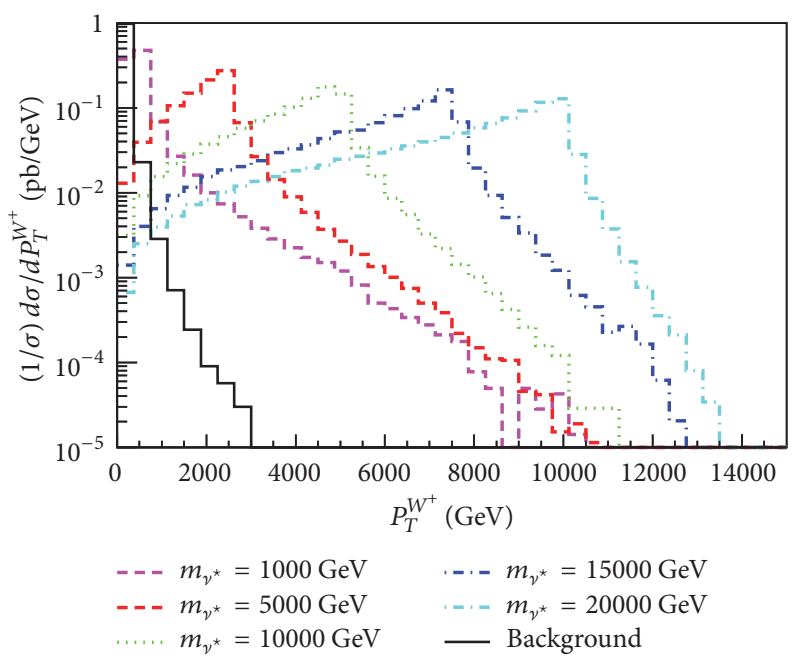

(a)

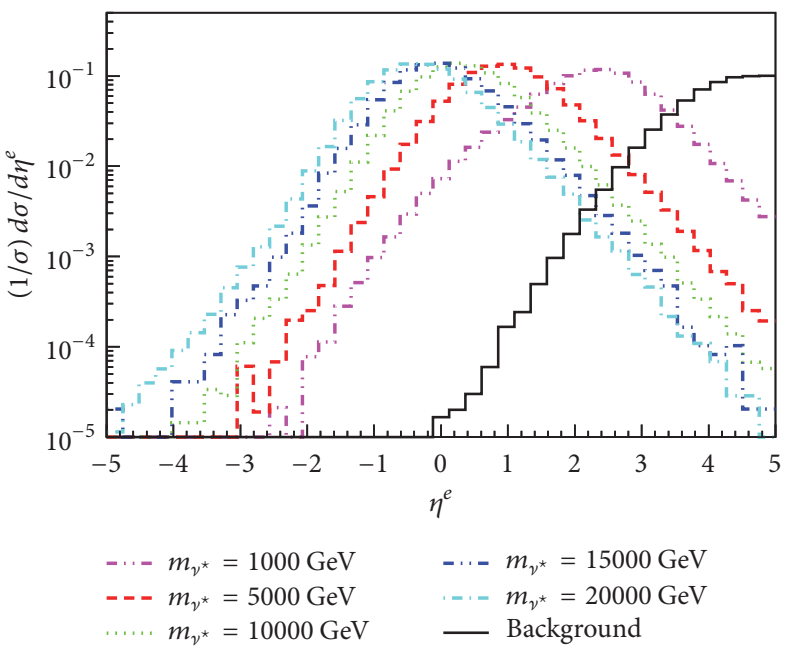

(b)

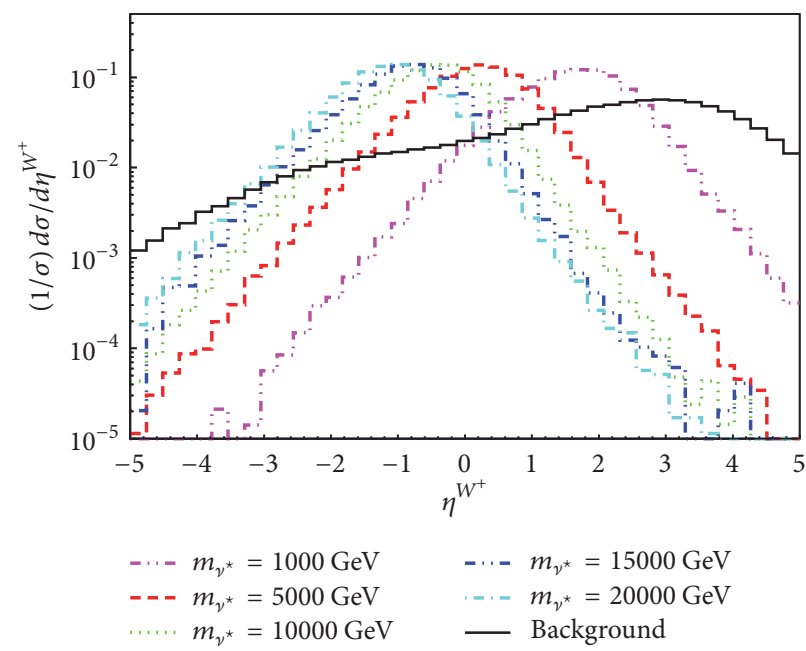

(c)

FIGURE 8: The normalized transverse momentum distributions of the final state $W^{+}$bosons (a), the normalized pseudorapidity distributions of the final state electrons (b), and the normalized pseudorapidity distributions of the final state $W^{+}$bosons (c) for $f=f^{\prime}=1$ and $\Lambda=m_{\gamma^{\star}}$ at the PWFA-LC $\otimes$ FCC collider.

TABLE 4: The statistical significance (SS) values and the cross sections of the excited neutrino signal and relevant background at the PWFA-LC $\otimes$ FCC collider with $\sqrt{s}=31.6 \mathrm{TeV}$ assuming the coupling $f=f^{\prime}=1$ and the energy scale $\Lambda=m_{v^{\star}}$.

\begin{tabular}{lcccc}
\hline Mass $(\mathrm{GeV})$ & $\sigma_{B}(\mathrm{pb})$ & $\sigma_{S+B}(\mathrm{pb})$ & $L_{\mathrm{int}}=1 \mathrm{fb}^{-1}$ & \multicolumn{2}{c}{$L_{\mathrm{int}}=10 \mathrm{fb}^{-1}$} \\
& & $\mathrm{SS}$ & 270.5 & 855.6 \\
2000 & $1.16 \times 10^{-3}$ & $2.92 \times 10^{-1}$ & 87.6 & 277.2 \\
4000 & $1.03 \times 10^{-3}$ & $9.00 \times 10^{-2}$ & 29.7 & 93.9 \\
6000 & $6.30 \times 10^{-4}$ & $2.42 \times 10^{-2}$ & 11.8 & 37.5 \\
8000 & $3.59 \times 10^{-4}$ & $7.47 \times 10^{-3}$ & 5.3 & 17 \\
10000 & $1.78 \times 10^{-4}$ & $2.45 \times 10^{-3}$ & 2.8 & 9.1 \\
12000 & $6.55 \times 10^{-5}$ & $8.02 \times 10^{-4}$ & 1.5 & 4.9 \\
14000 & $2.27 \times 10^{-5}$ & $2.57 \times 10^{-4}$ & 0.7 & 2.4 \\
16000 & $7.71 \times 10^{-6}$ & $7.66 \times 10^{-5}$ & 0.3 & 1.1 \\
18000 & $2.51 \times 10^{-6}$ & $2.06 \times 10^{-5}$ & 0.1 & 0.4 \\
20000 & $7.42 \times 10^{-7}$ & $4.85 \times 10^{-6}$ & & \\
\hline
\end{tabular}


TABLE 5: The mass limits for the exclusion $(2 \sigma)$, the observation $(3 \sigma)$, and the discovery $(5 \sigma)$ of the excited neutrinos at the different $e p$ colliders assuming the coupling $f=f^{\prime}=1$ and the energy scale $\Lambda=m_{v^{\star}}$.

\begin{tabular}{lcccc}
\hline Colliders & $L_{\text {int }}\left(\mathrm{fb}^{-1}\right)$ & $2 \sigma(\mathrm{GeV})$ & $3 \sigma(\mathrm{GeV})$ & $5 \sigma(\mathrm{GeV})$ \\
\hline ERL60 $\otimes$ FCC & 100 & 2618 & 2547 & 6000 \\
\\
ILC $\otimes$ FCC & 10 & 6300 & 6790 & 5635 \\
& 100 & 7025 & 11850 & 6460 \\
PWFA-LC $\otimes$ FCC & 1 & 13050 & 15450 & 10200 \\
\end{tabular}

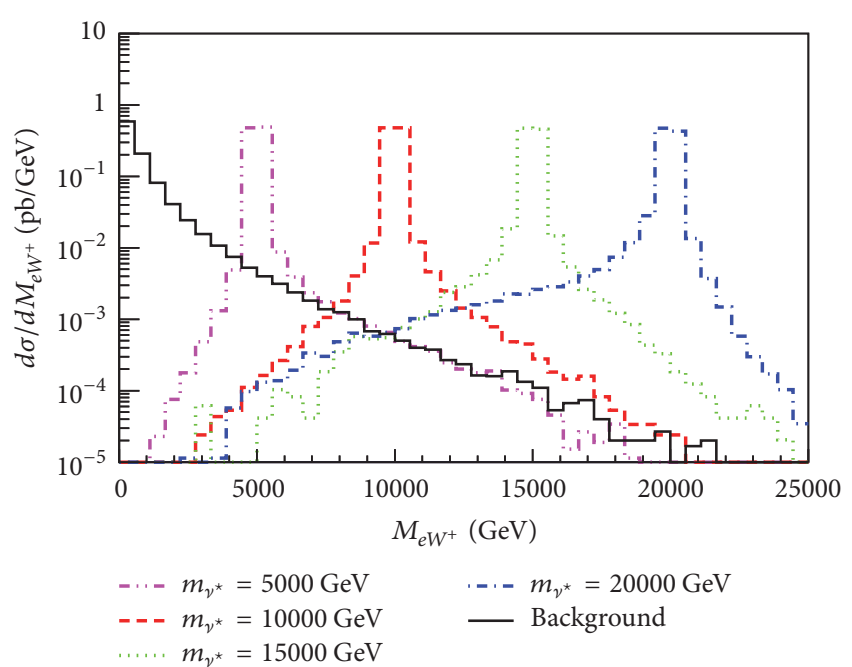

FIGURE 9: The invariant mass distributions of the excited neutrino signal and the corresponding background for $\Lambda=m_{v^{\star}}$ and $f=f^{\prime}=$ 1 at the PWFA-LC $\otimes$ FCC collider.

10200 and $13960 \mathrm{GeV}$ for the integrated luminosities of $L_{\text {int }}=$ $1 \mathrm{fb}^{-1}$ and $L_{\text {int }}=10 \mathrm{fb}^{-1}$, respectively.

\section{Conclusion}

This work has shown that the FCC-based ep colliders have a great potential for the excited neutrino searches. We give a realistic estimate for the excited neutrino signal and the corresponding background at three different colliders, namely, the $\mathrm{ERL} 60 \otimes \mathrm{FCC}(\sqrt{s}=3.46 \mathrm{TeV})$, the ILC $\otimes \mathrm{FCC}(\sqrt{s}=10 \mathrm{TeV})$, and the PWFA-LC $\otimes$ FCC $(\sqrt{s}=31.6 \mathrm{TeV})$. The simulations have been performed assuming the energy scale $\Lambda=m_{\gamma^{\star}}$ and the coupling parameter $f=f^{\prime}=1$. The mass limits for exclusion, observation, and discovery of the excited neutrinos at the three colliders are given in Table 5, for the different integrated luminosity values. As a result, these three FCCbased ep colliders offer the possibility of probing the excited neutrino over a very large mass range.

\section{Conflicts of Interest}

The author declares that he has no conflicts of interest.

\section{Acknowledgments}

The author is grateful to A. Ozansoy and S. O. Kara for useful discussions and model file supports. This work has been supported by the Scientific and Technological Research Council of Turkey (TUBITAK) under Grant no. 114F337.

\section{References}

[1] ATLAS Collaboration, "Observation of a new particle in the search for the Standard Model Higgs boson with the ATLAS detector at the LHC," Physics Letters B, vol. 716, no. 1, 2012.

[2] I. A. D'Souza and C. S. Kalman, PREONS: Models of Leptons, Quarks and Gauge Bosons as Composite Objects, World Scientific Publishing, 1992.

[3] J. H. Kühn, H. D. Tholl, and P. M. Zerwas, "Signals of excited quarks and leptons," Physics Letters B, vol. 158, no. 3, pp. 270275,1985

[4] U. Baur, M. Spira, and P. M. Zerwas, "Excited-quark and -lepton production at hadron colliders," Physical Review D: Particles, Fields, Gravitation and Cosmology, vol. 42, no. 3, pp. 815-824, 1990.

[5] Y. Tosa and R. E. Marshak, "Exotic fermions," Physical Review D: Particles, Fields, Gravitation and Cosmology, vol. 32, no. 3, pp. 774-780, 1985.

[6] L3 Collaboration, "Search for excited leptons at LEP," Physics Letters B, vol. 568, no. 1, 2003.

[7] H1 Collaboration, "Search for excited electrons in ep collisions at HERA," Physics Letters B, vol. 666, no. 2, 2008.

[8] D0 Collaboration, "Search for excited electrons in pp collision at s=1.96 TeV," Physical Review D, vol. 77, 2008.

[9] CMS Collaboration, "Search for excited leptons in protonproton collisions at s=8 TeV," Journal of High Energy Physics, vol. 125, 2016.

[10] ATLAS Collaboration, "Search for excited electrons and muons $\mathrm{s}=8 \mathrm{TeV}$ proton-proton collisions with the ATLAS detector," New Journal of Physics, vol. 15, no. 093011, 2013.

[11] O. Cakir, A. Yilmaz, and S. Sultansoy, "Single production of excited electrons at future e-e+, ep and pp colliders," Physical Review D, vol. 70, no. 075011, 2004.

[12] A. Ozansoy and A. A. Billur, "Search for excited electrons through $\gamma$ scattering," Physical Review D: Particles, Fields, Gravitation and Cosmology, vol. 86, no. 5, Article ID 055008, 2012.

[13] A. Caliskan, S. O. Kara, and A. Ozansoy, "Excited muon searches at the FCC-based muon-hadron colliders," Advances in High Energy Physics, vol. 2017, Article ID 1540243, 9 pages, 2017. 
[14] O. Cakir, I. T. Cakir, and Z. Kirca, "Single production of excited neutrinos at future e+e-, ep and pp colliders," Physical Review $D$, vol. 70, 2004.

[15] O. Cakir and A. Ozansoy, "Single production of excited spin-3/2 neutrinos at linear colliders," Physical Review D, vol. 79, 2009.

[16] M. Köksal, "Analysis of excited neutrinos at the CLIC," International Journal of Modern Physics A, vol. 29, no. 24, Article ID 1450138, 2014.

[17] A. Ozansoy, V. Ari, and V. Cetinkaya, "Search for spin-3/2 neutrinos at LHeC," Advances in High Energy Physics, vol. 2016, Article ID 1739027, 10 pages, 2016.

[18] C. Patrignani, K. Agashe, G. Aielli et al., "Review of particle physics," Chinese Physics C, vol. 40, 2016.

[19] FCC Project Web Page: https://fcc.web.cern.ch.

[20] M. Bicer et al., "First look at the physics case of TLEP," Journal of High Energy Physics, vol. 1401, p. 164, 2014.

[21] S. Sultansoy, A. Akay, Y. Acar, S. Beser, U. Kaya, and B. Oner, "FCC based lepton-hadron and photon-hadron colliders: luminosity and physics," in Proceedings of the Second Annual Meeting of the Future Collider Study (FCC Week '16), 2016.

[22] LHeC Project Web Page: http://lhec.web.cern.ch.

[23] C. Adolphsen, "The International Linear Collider Technical Design Report - Volume 3," in Proceedings of the The International Linear Collider Technical Design Report - Volume 3, p. physics, arXiv:1308.0494.

[24] E. Adli, J. Delahaye, S. Gessner et al., "A Beam Driven PlasmaWakefield Linear Collider: From Higgs Factory to Multi-TeV," Tech. Rep. SLAC-PUB-15426, 2013.

[25] Y. C. Acar et al., Main parameters of LCxFCC based electronproton colliders, arXiv:1602.03089 [hep-ph].

[26] Y. C. Acar et al., FCC based ep and $\mu$ p colliders, arXiv:1510.08284 [hep-ph].

[27] K. Hagiwara, D. Zeppenfeld, and S. Komamiya, "Excited lepton production at LEP and HERA," Zeitschrift für Physik C, vol. 29, no. 1, pp. 115-122, 1985.

[28] F. Boudjema, A. Djouadi, and J. L. Kneur, "Excited fermions at e+e- and ep colliders," Zeitschrift für Physik C Particles and Fields, vol. 57, no. 3, pp. 425-449, 1993.

[29] A. Belyaev, N. D. Christensen, and A. Pukhov, "CalcHEP 3.4 for collider physics within and beyond the standard model," Computer Physics Communications, vol. 184, no. 7, pp. 17291769, 2013.

[30] D. Stump, J. Huston, and J. Pumplin, "Inclusive jet production, parton distributions, and the search for new physics," Journal of High Energy Physics, vol. 2003, no. 10, article 046, 2003. 

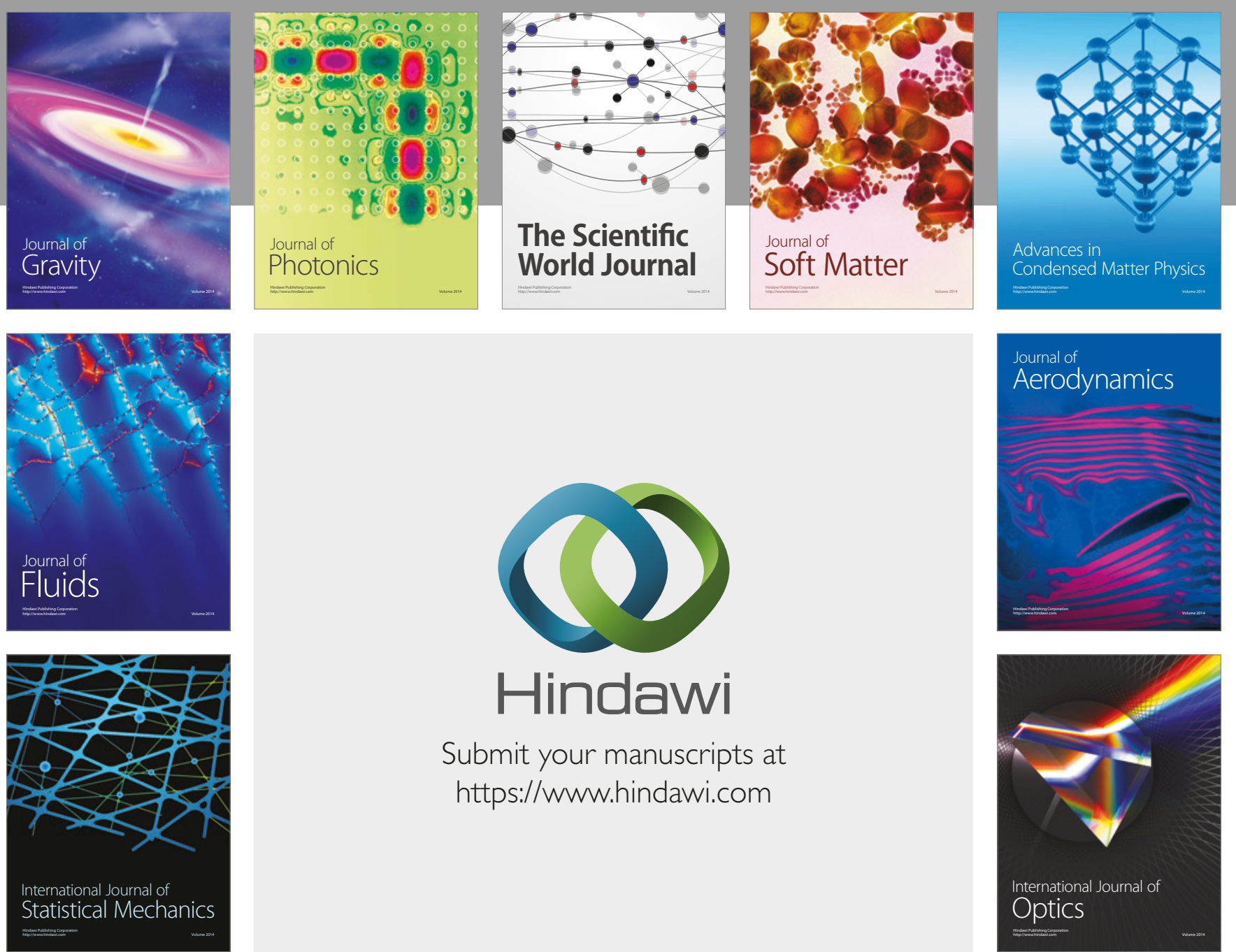

Submit your manuscripts at

https://www.hindawi.com
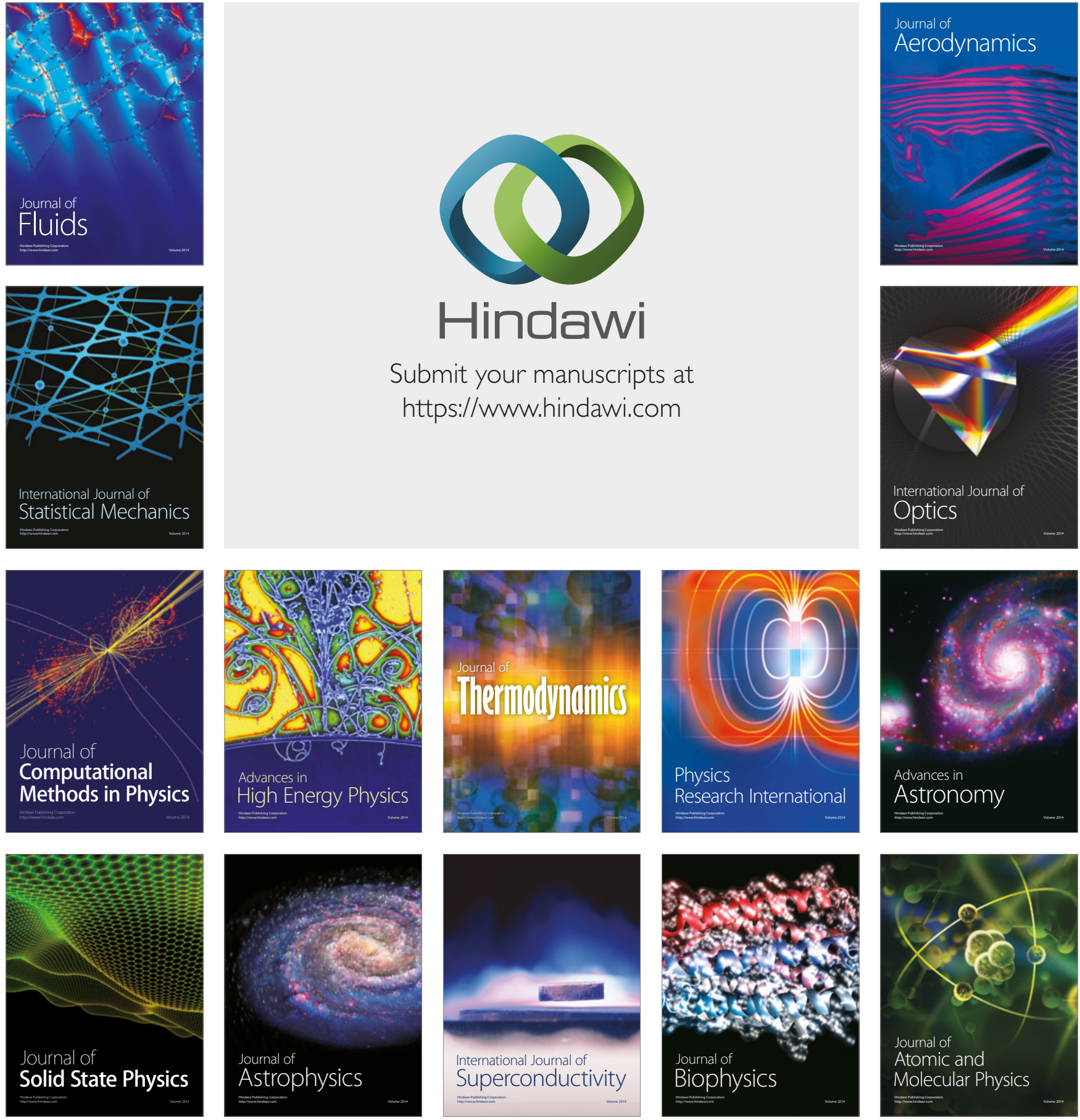